\title{
Transformer Condition Monitoring Technology Based on Surface Acoustic Wave Passive Wireless Sensing Antenna
}

\author{
Zhenwei $\mathrm{E}^{1, *}$, Dong $\mathrm{Fu}^{1}$, Zhengzhi $\mathrm{Yu}^{1}$, Yaqing $\mathrm{Hu}^{1}$ and $\mathrm{Yu} \mathrm{Nie}^{2}$ \\ ${ }^{1}$ State Grid Fushun Electric Power Supply Company, State Grid Liaoning Electric Power Supply Co., Ltd. 113008 Fushun, China \\ ${ }^{2}$ State Grid Liaoning Electric Power Supply Co., Ltd., 110004 Shenyang, China
}

\begin{abstract}
As the infrastructure for people's production and life, the stable operation of power facilities is very important. As a key equipment in the operation of power facilities, transformers have become important power equipment for the daily maintenance of the power sector. In the past, electric power operation and maintenance personnel mostly used on-site visual inspection to preliminarily judge whether the transformer is operating normally. The disadvantage of this method is inaccuracy. A transformer condition monitoring technology based on a surface acoustic wave passive wireless intelligent sensing system is proposed to overcome the above shortcomings. Its working mechanism is to monitor the oil level, oil temperature and external ambient temperature of the cooling oil in the transformer in real time. Then, the operating status can be determined. The operating data is transmitted to the control center through the $4 \mathrm{G}$ network to help the operation and maintenance personnel to centrally monitor the status of the transformer, and then provide a pre-alarm function for abnormal conditions of the transformer.
\end{abstract}

\section{Introduction}

With the development of the economy, the load has increased and transformer failures have occurred more and more frequently, which causes great troubles to the production and life of enterprises and residents. Since the transformer is directly connected to the load, the load imbalance problem cannot be solved, which causes the rise of the zero sequence current, the heating of the iron core, and the excessive oil temperature [1]. At the same time, the geographical distribution of distribution transformers is very scattered, and it is difficult to find problems such as temperature rise, oil temperature rise, and low insulating oil level in a timely manner by relying on current inspection methods.

Therefore, it is necessary to transform the distribution transformer. This paper establishes a comprehensive online transformer status monitoring technology integrating load analysis, transformer lowvoltage outlet temperature, transformer oil temperature and oil level.

Transformer equipment monitoring technology is widely studied by scholars, such as, infrared temperature measurement, active wireless temperature measurement, and distributed optical fiber temperature measurement. Infrared temperature measurement is currently one of the most widely used temperature measurement methods for electric power [2]. It is suitable for live testing of the surface temperature and point temperature of the equipment within the visible range, but its test results are greatly affected by environmental conditions and are suitable for inspections and inspections [3-5]. On-line detection: Active wireless temperature measurement schemes now generally use batteries or current transformers (CT) to supply power to the temperature measurement chip, and the sensing distance is very long. However, in harsh environments such as high temperature and strong electromagnetic fields, there are problems with the life of batteries and electronic components. The active sensor that adopts the CT power-taking method, because the $\mathrm{CT}$ power-taking coil has installation location requirements, it can not supply power even in the state of line failure, and its application also has great limitations. Optical fiber temperature measurement is a wired temperature measurement method, and the optical fiber or its sheath on the primary side of the high voltage measurement has a problem of insulation to the ground. At the same time, the optical fiber has the characteristics of being easy to break and break, and the cost of optical fiber sensor equipment is relatively high, which is not suitable for application in complex and scattered distribution networks.

The battery wireless sensor realizes the wireless function by adding a battery to the traditional sensor, which is the simplest to achieve, but the battery needs to be replaced after a certain period of time [6]. The battery is also sensitive to temperature, therefore, the performance of the low temperature battery decreases.

The wireless sensor of power-taking technology generally uses a current induction coil to obtain energy through electromagnetic induction in a fixed ratio, and it is no longer necessary to replace the battery for longterm work. However, it must have a closed circuit, so there are certain installation requirements during

*Corresponding author: 124358916@qq.com 
installation [7]. It must be surrounded by the joint. The installation volume is generally large. At the same time, the closed circuit is prone to eddy current heating under high current, which becomes a safety hazard. Moreover, the low-voltage load generally changes greatly, and the repeated large-scale changes of the current will also cause an impact on the power-taking device and accelerate the damage.

The passive wireless temperature measurement device based on surface acoustic wave technology uses piezoelectric technology to realize the intrinsically passive and natural wireless of the sensor without the need for batteries or power-taking devices. The sensor can be installed to the low pressure joint by simple bolt fastening. The performance and life of the sensor are not affected by temperature and load current. At the same time, the sensor is intrinsically safe for the equipment. Even in the worst case, if the sensor is damaged, it will not affect the normal operation of the primary equipment.

\section{The principle of surface acoustic wave wireless passive temperature measurement technology}

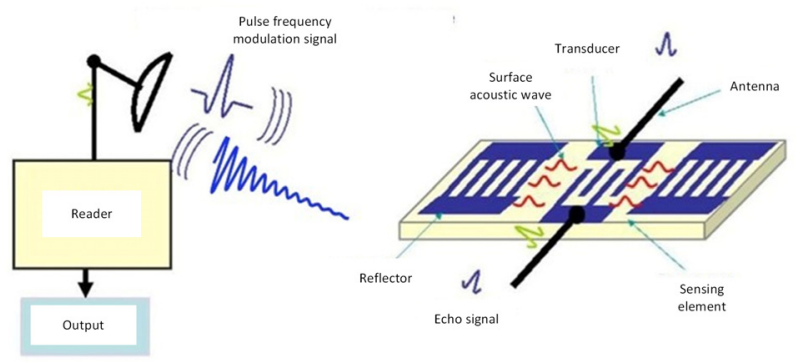

Figure 1 Schematic diagram of wireless sensing system

In the surface acoustic wave resonator (SAWR) wireless sensor system shown in Figure 1, the reader transmits narrowband electromagnetic waves through the reader antenna. The electromagnetic waves are received by the sensor antenna and excite a single-port SAWR made by piezoelectric technology. Through the inverse piezoelectric effect, the interdigital transducer (IDT) converts the electromagnetic wave received by the sensor antenna into a narrow-band surface acoustic wave. The actual resonant frequency of a single-port SAWR is determined by the structure of the resonant cavity and the environmental impact of the substrate (such as temperature, strain, etc.). When the excitation disappears, the surface acoustic waves of each frequency component in the band will freely decay and oscillate with different time constants. Only the electromagnetic wave with the same frequency as the SAWR natural resonant frequency lasts the longest. IDT uses the piezoelectric effect to convert the surface acoustic waves into electromagnetic waves and radiate them from the antenna. The reader estimates the natural resonance frequency of SAWR after receiving the attenuated oscillating electromagnetic wave affected by temperature, and then wireless temperature measurement can be realized.
The frequency-temperature characteristics of the single-port SAWR used for temperature sensing characterize the tendency of the natural frequency of SAWR to change with temperature. Through the analysis of the frequency-temperature characteristics of the resonator, information such as the sensitivity and occupied bandwidth of the SAW temperature sensor can be obtained, so as to guide the design of the reader and determine some reader design indicators.

When a narrow-band pulse signal is used to excite SAWR, the echo signal obtained is an attenuated and oscillating sinusoidal signal. The energy and duration of the signal are related to the quality factor ( $Q$ value) of the sensor. Only when the excitation frequency is the same as the SAWR resonance frequency, the echo energy reaches the maximum and the duration is the longest. When there is a certain gap between the excitation frequency and the natural frequency of the resonator, the energy and duration will be reduced accordingly.

\section{Antenna system design}

\subsection{Antenna plan development}

Since the oil level gauge itself is made of metal, and the antenna system requires a certain metal clearance area or adopts a metal-resistant design scheme, the maximum performance of the antenna can be exerted. Due to the limitation of actual conditions, there is not enough headroom area around the antenna, and the operating frequency of the system is $433 \mathrm{MHz}$. From Equation 1, the wavelength is about $0.697 \mathrm{~m}$. According to antenna theory, when there are metal objects around the antenna, the performance of the antenna will be greatly affected. interference. Therefore, when designing the sensor antenna of this monitoring system, it is necessary to focus on the balance between antenna performance and operating distance.

$$
\lambda=c / f
$$

where $\lambda$ is the wavelength, $c$ is the speed of light, and $f$ is the operating frequency.

This system needs to detect three parameters, namely oil temperature, oil level and ambient temperature. Two sets of temperature sensor subsystems need to be assembled, which are divided into oil temperature sensor subsystem and ambient temperature and oil level sensor subsystem. The two sensors use frequency division. Technology to ensure that there will be no serious cochannel interference between the two. Emphasize on the working principle of the ambient temperature oil level sensor subsystem: through a special antenna circuit design, when the monitoring system is working normally, the ambient temperature data is transmitted back to the system, which is regarded as normal oil level. When the oil level is lower than a certain value. The antenna circuit is disconnected and the ambient temperature data disappears, which is regarded as abnormal oil level. The antenna parts of the two sets of sensor subsystems have certain differences in appearance, but the requirements 
of working principle, simulation target and measured performance are the same.

Considering the characteristics of easy disassembly and installation, low wind resistance, anti-aging, long life, and waterproof of actual field installation, the reader antenna of the monitoring system adopts a magnetic copper rod antenna scheme. The reader antenna is firmly attracted to the transformer cover through a strong magnet, and kept at a distance of $30 \sim 50 \mathrm{~cm}$ from the oil level gauge under the premise of maintaining a certain safety interval. The reader antenna is an omnidirectional monopole antenna, and the antenna radiator is a slender cylindrical metal copper rod (diameter $12 \mathrm{~mm}$, length $170 \mathrm{~mm}$ ), which has excellent low wind resistance, waterproof and anti-aging properties. The reader antenna is shown in Figure 2. The reader antenna is connected to the reader through the feeder.

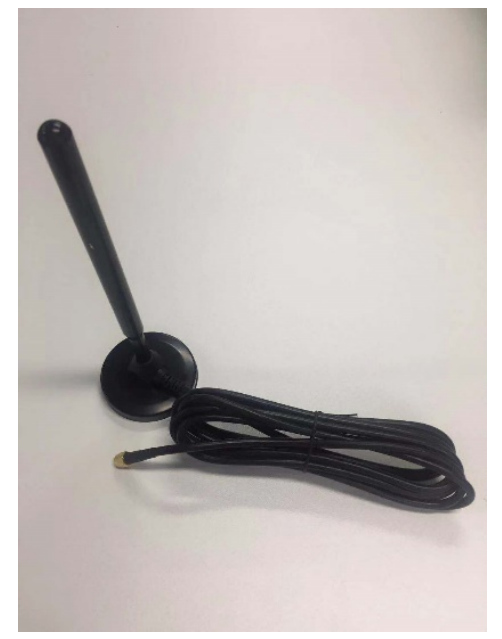

Figure 2 Reader antenna

\subsection{The basic theory of Planar Inverted-F Antenna}

The basic structure of Planar Inverted-F Antenna (PIFA) includes four parts: ground plane, radiating element, short-circuit metal sheet and coaxial feeder. Its typical basic structure is shown in Figure 3.

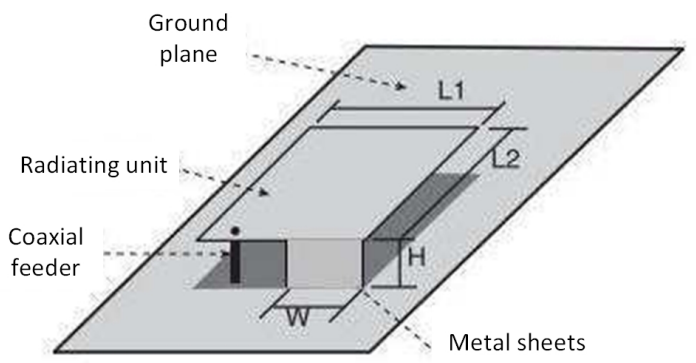

Figure 3 Typical structure of PIFA antenna

According to the PIFA antenna theory, the metal sheet width $L_{1}$, length $L_{2}$, short-circuit metal sheet width $W$, and radiating sheet height $H$ shown in Figure 3 are closely related to the resonant frequency of the antenna. By adjusting the dimensions of $L_{1}, L_{2}, H$, and $W$, an antenna that works in any frequency band and impedance can be obtained. When the width $W$ of the short-circuit metal sheet is equal to the width $L_{1}$ of the radiating metal sheet, that is, when $\frac{W}{L_{1}}=1$, there is

$$
\begin{gathered}
\frac{\lambda}{4}=H+L_{2} \\
f_{r}=\frac{c}{4\left(H+L_{2}\right)}
\end{gathered}
$$

When the width of the short-circuit metal sheet $\mathrm{W}=0$, there are:

$$
\begin{gathered}
\frac{\lambda}{4}=H+L_{1}+L_{2} \\
f_{r}=\frac{c}{4\left(H+L_{1}+L_{2}\right)}
\end{gathered}
$$

In the formula, $\lambda$ represents the resonance wavelength, $f_{r}$ represents the resonance frequency, and $c$ represents the speed of light.

For short-circuit metal sheets of any width $\mathrm{W}$, the resonant frequency can be calculated by the following formula:

$$
f_{r}=r^{k} f_{1}+\left(1-r^{k}\right) f_{2}
$$

where

$$
r=\frac{w}{L_{1}}, k=\frac{L_{1}}{L_{2}}, f_{1}=\frac{c}{4\left(H+L_{2}\right)}, f_{2}=\frac{c}{4\left(H+L_{1}+L_{2}\right)} .
$$

\section{Analysis of simulation results}

Based on equations (2), (3), (4) and (5), the antenna model is established in the HFSS software, and the setting of the antenna size model parameters refers to the initial simulation parameters calculated according to the formula. in Figure 4 and Figure 5. The antenna size model parameter setting the initial simulation parameters are selected. After multiple rounds of simulation and parameter optimization, when the short-circuit metal sheet width $W=1.5 \mathrm{~mm}$, the radiation metal sheet width $L_{1}=10 \mathrm{~mm}$, the length $L_{2}=112 \mathrm{~mm}$, and the height $H=5 \mathrm{~mm}$, the simulation results are ideal. It can be seen from Figure 6 and Figure 7 that when the operating frequency is $439 \mathrm{MHz}$, the $\mathrm{S}$ parameter $S_{11}=-19 \mathrm{~dB}$ and the gain Gain $=9.4 \mathrm{~dB}$, the performance effect meets the predetermined target value.

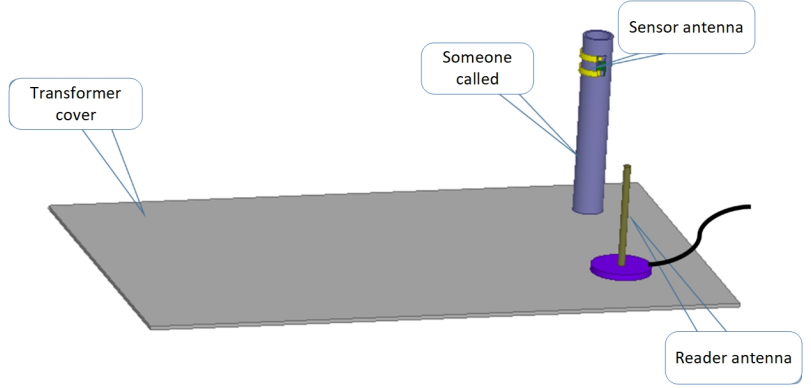

Figure 4 Software simulation model 


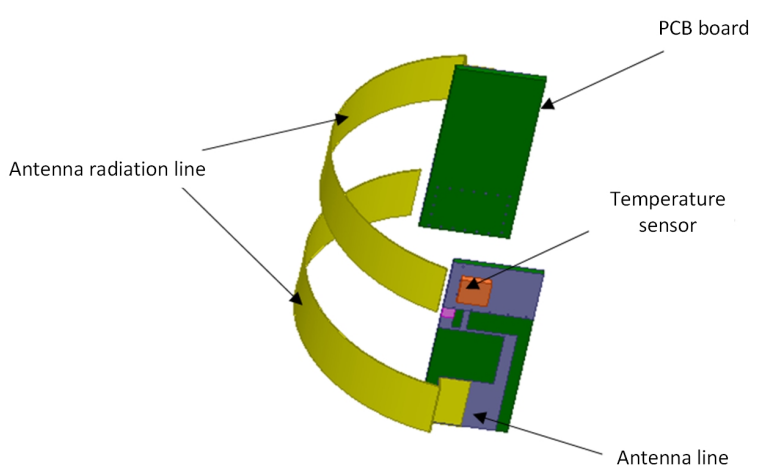

Figure 5 Simulation model of sensor antenna

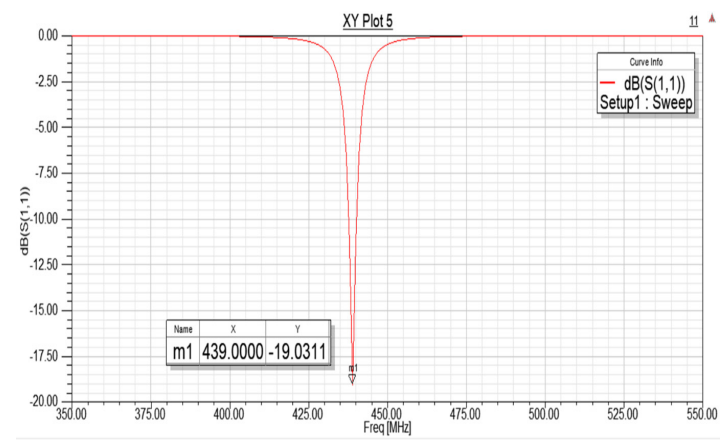

Figure $6 \mathrm{~S}$ parameter of sensor antenna

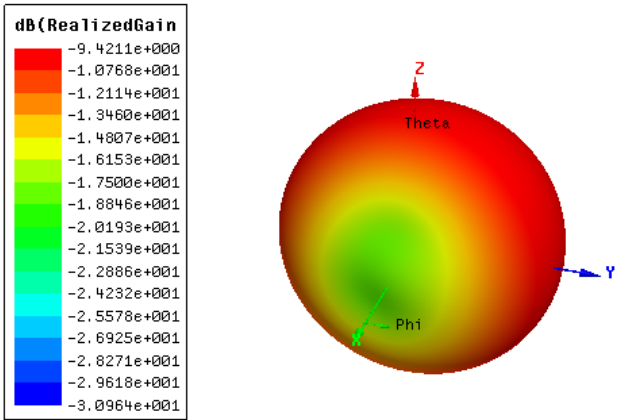

Figure 7 Sensor antenna gain

At the same time, some regular experience is summarized in the simulation analysis, for example, the increase of the height $H$ can increase the gain value and reduce the resonance frequency. The increase in the width $W$ of the short-circuit metal sheet increases the resonance frequency.

\section{Conclusion}

In this paper, research and application of online monitoring technology for distribution transformers are carried out. The passive and wireless sensing scheme of surface acoustic wave technology was adopted to realize the online monitoring and early warning of the operation status of distribution transformers. The reliable operation of the transformer is ensured.

\section{References}

1. G Wang, Y Liu, X Chen, et al. Power transformer fault diagnosis system based on Internet of Things[J]. EURASIP Journal on Wireless Communications and Networking, 2021, 2021(1).

2. C Bengtsson. Status and trends in transformer monitoring $[\mathrm{J}]$. IEEE Transactions on Power Delivery, 1996, 11(3): pp.1379-1384.

Q Sun, W Qian, W Nan. Design of Distribution Transformer Monitoring System Based on LPC2103[J]. Journal of Convergence Information Technology, 2012, 7(21): pp.120-126.

3. D Peharda, I Ivankovi, N Jaman . Using Data from SCADA for Centralized Transformer Monitoring Applications[J]. Procedia Engineering, 2017, 202: pp.65-75.

4. A Raghavan, P Kiesel, M Tee Pe, et al. Low-cost embedded optical sensing systems for distribution transformer monitoring[J]. IEEE Transactions on Power Delivery, 2020, 99: pp.1-1.

5. V. Catterso, S. Mcarthur, G. Moss. Online Conditional Anomaly Detection in Multivariate Data for Transformer Monitoring[J]. IEEE Transactions on Power Delivery, 2010, 25(4): pp.2556-2564.

6. B. Garcia, J. Burgos, A. Alonso, et al. A moisturein-oil model for power transformer monitoring - Part II: Experimental verification[J]. IEEE Transactions on Power Delivery, 2005, 20(2): pp.1423-1429. 